\title{
Representaciones sociales y violencia de género: caso resguardo indigena Cañamomo-Lomaprieta, Riosucio, Caldas
}

\section{Social representations and gender violence: a case of indigenous reservation Cañamomo, Lomaprieta, Riosucio Caldas}

\author{
Jonnathan Harvey Narváez*, Carol Dayana Jurado“*
}

Universidad de Nariño

Recibido: 15 de mayo de 2020-Aceptado: 22 de julio de 2021-Publicado: 1 de enero de 2022

Forma de citar este artículo en APA:

Narváez, J. H., \& Jurado, C. (2022). Representaciones sociales y violencia de género: caso resguardo indígena Cañamomo, Lomaprieta, Riosucio,

Caldas. Revista Colombiana de Ciencias Sociales, 13(1), 349-370. https://doi.org/10.21501/22161201.3621

\section{Resumen}

En este artículo se presenta un análisis de las representaciones sociales frente a la violencia contra la mujer en el resguardo indígena Cañamomo Lomaprieta, en Riosucio, Caldas. Dicho análisis se desarrolla a partir de la propuesta de Abric (2001), quien establece que las representaciones se organizan estructuralmente en un núcleo central y unos elementos periféricos, y que es la identificación de esta estructura la base para

Doctor en Ciencias de la Educación Universidad de Cuauhtémoc. Docente Investigador Departamento de Psicología Universidad de Nariño. Integrante del grupo de Investigación Libres Pensadores, Pasto-Colombia. Contacto: jonnathanharnarvaez@gmail.com. ORCID https://orcid.org/0000-0002-3023-5156, https://scholar.google.es/citations?user=kg-QxfwAAAAJ\&hl=es “Psicóloga Universidad de Nariño. Integrante del grupo de Investigación Libres Pensadores, Pasto-Colombia. Contacto: karoljurado32@gmail.com. ORCID https://orcid.org/0000-0002-75910450 
analizar y comprender la representación social. La investigación se realizó bajo el paradigma cualitativo, siguiendo un enfoque histórico hermenéutico y un tipo de estudio de teoría fundamentada, con 60 mujeres entre los 20 y 70 años, pertenecientes a la asociación de mujeres del territorio. Los resultados del estudio indican 4 representaciones sociales: toda forma de violencia de género nace a partir de la no valoración de las mujeres en el territorio, la violencia de género se forja en el hogar, ante la violencia de género contra la mujer en el territorio es más oportuno quedarse callada y las mujeres que son permisivas con la violencia son culpables de esta.

\section{Palabras clave}

Representaciones sociales; Violencia de género; Mujer indígena; Interseccionalidad; Interculturalidad.

\section{Abstract}

This article presents an analysis of social representations against violence against women in the indigenous reservation Cañamomo Lomaprieta, Riosucio Caldas, is developed from the proposal of Abric (2001), who establishes that these are structurally organized in a central nucleus and peripheral elements and that it is the identification of this structure the basis for analyzing and understanding social representation. The research was carried out under the qualitative paradigm, following a historical hermeneutic approach and a type of study of grounded theory, with 60 women between 20 and 70 years old, belonging to the association of women of the territory. The results of the study indicate four social representations: All forms of gender violence arise from the non-valuation of women in the territory, gender violence is forged in the home, in the face of gender violence against women in the territory it is more opportune to remain silent and women who are permissive with violence are guilty of it.

\section{Keywords}

Social representations; Gender violence; Indigenous woman; Intersectionality; Interculturality. 


\section{Introducción}

La violencia contra la mujer es un tema central en el debate social y político, puesto que su pilar se encuentra en los valores y normas arraigados en estructuras sociales que agravan las desigualdades entre lo masculino y lo femenino, y que atentan contra los derechos humanos de las mujeres a través de prácticas cotidianas que se reproducen en las formas de socialización (CIDH, 2017). En Colombia, la violencia de género contra la mujer es definida por la Ley 1257 de 2008 como:

cualquier acción u omisión, que le cause muerte, daño o sufrimiento físico, sexual, psicológico, económico o patrimonial por su condición de mujer, así como las amenazas de tales actos, la coacción o la privación arbitraria de la libertad, bien sea que se presente en el ámbito público o en el privado. (p. 1)

En el caso de las mujeres indígenas, este tipo de violencia se define centralmente en la relación entre la discriminación de género dentro y fuera del contexto territorial, los aspectos propios de la identidad étnica y los sistemas de dominación social, haciendo necesario que al hablar de violencia contra la mujer indígena se reconozcan, primeramente, los múltiples elementos socioculturales que en interacción con el género producen en la cotidianidad formas particulares de abuso asumidas desde los referentes simbólicos con los que se construye e interpreta la realidad y que refuerzan las estructuras tradicionales que la permiten y la invisibilizan (FIMI, 2006). Este abordaje de la violencia de género en el contexto indígena desde un enfoque de interseccionalidad permite reconocer que en la violencia de género contra la mujer juega un papel importante la relación entre el carácter étnico, el género y la condición social. Por lo tanto, aunque se alude a una definición generalizada de violencia de género, no todas las mujeres viven esta realidad de la misma manera, debido a que en ella convergen múltiples y simultáneos sistemas de opresión que operan a partir de categorías definidas y reproducidas socialmente, por lo que es necesario entender el contexto desde el cual se construye y entiende esta realidad (Juárez-Moreno et al., 2021).

En el contexto de las mujeres indígenas embera chamí, la violencia de género se vive a través de un conjunto de prácticas y relaciones asimétricas entre lo femenino y lo masculino que perpetúan un lugar de subordinación para la mujer en los ámbitos público y privado, y que adquiere manifestaciones que atentan contra sus derechos (Quintero \& García, 2021).

Este escenario representa para las mujeres del resguardo de origen colonial, Cañamomo Lomaprieta, del municipio de Riosucio, Caldas, una preocupación creciente debido al aumento en los indicadores de maltrato al interior del territorio. Por esta razón, se planteó la necesidad de realizar una articulación entre la academia y la gobernanza indígena, a fin de reconocer todos aquellos procesos de interacción comunitaria que ratifican la vulneración de los derechos de las mujeres como un actuar naturalizado. Para ello, se tomó como referente teórico la perspectiva de Jean Claude Abric (2001) sobre las representaciones sociales. Este autor basa su planteamiento en la premisa de Moscovici, quien señala que la sociedad, los individuos y las representaciones son 
un conjunto de construcciones sociales que permiten dar sentido a la realidad vivida. Para Moscovici, las representaciones sociales son un sistema cognitivo, con un lenguaje y lógica propios, orientado a organizar la realidad de acuerdo con sistemas de valores, ideas y prácticas que posibilitan a los individuos orientarse en su mundo material y social, los cuales facilitan la elaboración de la comunicación y los comportamientos individuales, dando paso a un sentido de conciencia colectiva que tiene como objetivo crear y recrear una identidad social (Mora, 2002).

Como fenómeno colectivo, las representaciones sociales permiten edificar y transformar la realidad a partir de construcciones simbólicas que se crean y recrean en el curso de las interacciones sociales a partir de dos procesos: el primero, reconocido como anclaje, permite transformar algo extraño en algo familiar, insertándolo en la dinámica social en un marco de lo conocido y preexistente; el segundo, denominado objetivación, busca transformar algo abstracto en algo concreto (Ceballos \& González, 2018). Abric (2001) organiza este sistema de información en una doble estructura, un núcleo central que ordena los elementos cognitivos relativos al objeto de representación que, al ser más estables, rígidos y consensuados colectivamente, aseguran la permanencia de la representación, evocan una gran resistencia al cambio y brindan una homogeneidad e identidad al grupo, organizando de manera coherente y con significación global la representación social; y un sistema periférico de la representación, conformado por un conjunto de elementos dinámicos, flexibles e individualizados que permiten que la representación se adapte a los contextos sociales a través de una relativa armonía en las situaciones y prácticas concretas de la cotidianidad, y que la representación funcione como tabla de decodificación de las situaciones (Rateau \& Mónaco, 2013).

Para el estudio de estas representaciones, Abric (2001) propone 3 dimensiones de análisis: la primera, referente a un campo de información donde se asientan los conocimientos que tiene el grupo frente a un acontecimiento social, que hace referencia a la cantidad, calidad, veracidad o trivialidad de este, lo cual implica la riqueza de datos o explicaciones que sobre la realidad se forman los individuos en sus interacciones sociales; la segunda dimensión, conocida como actitud, se refiere a la orientación favorable o desfavorable con relación al objeto de representación social, y encierra el componente más aparente y conductual de la representación, por lo que es la dimensión más estable de la misma; finalmente, el campo de representación permite visualizar el carácter del contenido, sus características cualitativas o imaginativas, integrándolas con un nuevo nivel de organización respecto a sus fuentes inmediatas -este debe analizarse en función de la totalidad del discurso sobre un objeto y no solo sobre un párrafo o frase, puesto que a partir de él se define la composición de los elementos, clasificando los contenidos de la representación en el núcleo central y el sistema periférico (Nuño, 2004)-. Estas dimensiones que conforman la estructura de sentido de las representaciones sociales no presentan un orden jerarquizado, sin embargo, permiten comprender la significación individual y colectiva de la representación. 
En efecto, el presente artículo presenta un análisis de las representaciones sociales frente a la violencia de género contra la mujer en el resguardo indígena Cañamomo Lomaprieta del municipio de Riosucio, Caldas, en concordancia con los planteamientos de Abric (2001), a partir de un ejercicio investigativo que posibilitó la apertura de un marco de conocimiento que en lo contextual brinda herramientas a la comunidad de Cañamomo para diseñar e implementar procesos en pro de la disminución de la violencia contra la mujer, desde acciones estratégicas consolidadas en el plan de vida de sus comunidades; y que en lo académico se presenta como una oportunidad de avance de la perspectiva interdisciplinar del saber psicológico, a través de una praxis que reivindica la aproximación real a la comunidad en un campo de intervención de gran relevancia actual en un doble sentido: el de la representación en sí misma, como objeto de estudio de la psicología social, y el de la violencia de género contra la mujer indígena, como una problemática estructural, histórica y social que debe comprenderse desde el campo de sentido y significado de la cognición social.

\section{Metodologia}

El estudio tiene por objetivo analizar las representaciones sociales frente a la violencia contra la mujer en el resguardo indígena Cañamomo Lomaprieta, en Riosucio, Caldas, y se orientó de acuerdo con los planteamientos del paradigma cualitativo, tomando como eje central la exploración de la naturaleza profunda de las realidades sociales y la comprensión de las conexiones del individuo con la vida cultural y social (Portilla et al., 2014), lo que favorece el reconocimiento de las experiencias subjetivas e intersubjetivas que permiten develar los significados atribuidos a la realidad y describir los procesos que generan una construcción social de la violencia contra la mujer en el resguardo. Todo ello, bajo el principio fundamental del respeto por el contexto natural de los sujetos de estudio, buscando reconocer los aspectos subjetivos asociados a la cotidianidad de las mujeres frente a la violencia de género. A su vez, se atendieron las orientaciones epistemológicas del enfoque histórico hermenéutico, donde se privilegió la comprensión, significatividad y relevancia social y cultural que adquieren las representaciones sociales frente a la violencia contra la mujer al interior del resguardo y las prácticas asociadas a esta, partiendo desde el conocimiento de su contenido, la relación entre sus elementos y el significado social dado bajo las condiciones históricas, culturales e individuales (Ortiz, 2012).

El tipo de estudio fue la teoría fundamentada, la cual, según Glaser-Holton (2004), constituye una metodología general para el análisis de datos cualitativos mediante el uso sistemático de un conjunto de métodos que buscan generar una teoría inductiva en torno a un área específica del campo de representación humano. Teniendo en cuenta el carácter subjetivo de la información recolectada, resultó pertinente realizar un proceso de descripción, comparación y conceptualización 
de los datos, de modo que, a través del método comparativo constante como principal procedimiento de la teoría fundamentada, se logró recoger y organizar sistemáticamente la información, para describir el contenido, develar la estructura y la jerarquía que se establece entre los elementos constitutivos centrales y periféricos de la representación, permitiendo una aproximación al sentido y significado que tienen para los sujetos la violencia contra la mujer como objeto de representación (Restrepo, 2013).

\section{Participantes}

La investigación se desarrolló con 60 mujeres entre los 20 y 70 años, representantes de la asociación de mujeres del resguardo indígena Cañamomo Lomaprieta, tomando como criterio de inclusión la aceptación voluntaria para participar en el estudio manifestada a través de la firma del consentimiento informado. Como criterios de inclusión se consideraron el ser parte del resguardo indígena y haber vivido al menos los últimos 10 años en la comunidad. El muestreo fue no probabilístico de tipo intencional bajo el sistema de bola de nieve con invitación directa.

\section{Técnicas de recolección de información}

Se utilizaron 3 técnicas para la recolección de la información. La entrevista semiestructurada, que posibilitó el acceso al contenido de la representación social individual de las mujeres, a través de un espacio de diálogo y comprensión de sus perspectivas, creencias, valores, opiniones, conocimientos y sentires (Cuevas, 2016). Con ella se favoreció un acercamiento íntimo, flexible y abierto a los elementos constitutivos de la representación frente a la violencia contra la mujer. Seguidamente, se desarrolló una cartografía social a través de la cual se reconocen los elementos constitutivos de las representaciones sociales frente a la violencia contra la mujer dentro de la comunidad y su influencia en el día a día, a partir de una reflexión crítica generada por las participantes acerca de su cuerpo, su espacio y las creencias individuales y comunitarias en torno al fenómeno de estudio, tomando como punto de diálogo las vivencias. De esta manera, se dio paso a un reconocimiento de los significados propios y colectivos otorgados a la violencia contra la mujer en el territorio (Barragán, 2015). Finalmente, se realizaron grupos focales en los que se generaron espacios de opinión entre las participantes e investigadores, lo que permitió captar el sentir, pensar y vivir de las mujeres en su pluralidad y variedad de actitudes, experiencias y creencias (Hamui \& Varela, 2013). La técnica se desarrolló mediante la formulación de preguntas orientadas a hacer un cuestionamiento del núcleo central, previamente identificado, de esta manera se hizo un contraste entre aspectos recabados a través de la entrevista semiestructurada y la cartografía social. 


\section{Procedimiento}

En la primera fase de la investigación se realiza un proceso reflexivo y de diseño en el que se define el objeto de representación a ser indagado en la investigación teniendo en cuenta el contexto cultural de la comunidad donde se desarrolla, para lo cual se enfatiza en el diálogo con las autoridades indígenas y la asociación de mujeres y se hace una revisión consciente del plan de vida de la comunidad. Seguidamente, se planifica la investigación y se establece una guía para la actuación en el trabajo de campo y el plan de análisis de datos. En la fase dos de la investigación se realiza el trabajo de campo, tomando como primera medida el acercamiento informal con las participantes y la observación del contexto; posteriormente se realiza la aplicación de los instrumentos de recolección de la información, mismos que fueron revisados y aprobados previamente por la mesa técnica de la asociación de mujeres del resguardo, teniendo en cuenta que, al tratarse de una comunidad indígena, esta cuenta con criterios y normas propias que deben ser respetadas por agentes externos; una vez efectuado el trabajo de campo, se ejecuta de manera progresiva y sistemática el plan de análisis. Para terminar, se construye el informe final del estudio, el cual es presentado a la comunidad en un ejercicio reflexivo de devolución de emergentes.

\section{Plan de análisis de la información}

El análisis de la información se realizó a través de 4 etapas. Primeramente, la identificación del contenido de la representación, lo cual corresponde a un momento descriptivo del análisis en el que se realizó la codificación abierta de la información con el objeto de identificar y describir las categorías emergentes respecto a la violencia contra la mujer. Para llevar a cabo esta tarea se hizo una fragmentación de la información, examinando línea por línea las respuestas dadas. Seguidamente, esta información fragmentada se conceptualizó y nominó, es decir, se estableció un código verbal para interpretarla y, finalmente, se categorizaron los conceptos y organizaron jerárquicamente.

Se pasó luego a la segunda etapa, el momento relacional del análisis en el que se establecen las relaciones entre los elementos, su importancia relativa y su jerarquía, con el propósito de: (a) indagar por la organización del contenido en un sistema de categorías y subcategorías que forman el esqueleto de la representación; (b) identificar los lazos, las relaciones y las jerarquías entre los elementos que componen la representación social y; (c) poner en evidencia los elementos centrales que organizan y le dan significación a la representación. Para esto, se realizó, en un primer momento, la codificación axial de la información, a partir de la cual se desarrollaron categorías conceptuales, y la codificación selectiva de la información, consistente en la reconstrucción teó- 
rica de las relaciones entre las categorías encontradas en el trascurso del estudio; el segundo momento fue la redacción de un conjunto de postulados interpretativos producto de la integración de las categorías deductivas y emergentes.

En la tercera etapa del análisis se realizó la determinación y control del núcleo central, buscando identificar la estructura y dinámica de cada representación social desde el núcleo de relaciones integradoras con el sistema periférico, para ello se aplicó en el espacio de grupos focales la técnica de cuestionamiento de núcleo central.

Finalmente, en la cuarta etapa se hizo un análisis de la argumentación a través de un acercamiento al funcionamiento contextualizado de las representaciones sociales, lo cual permitió develar los lazos entre las representaciones sociales y el sistema de valores propios de la comunidad (Restrepo, 2013).

\section{Resultados}

A continuación, se muestran los resultados obtenidos, planteando el núcleo central, seguido de los elementos periféricos y la relación entre estos elementos, con la intención de hacer una aproximación más global de unas representaciones sociales que se mantienen ancladas a las prácticas y lazos sociales en la comunidad y generan un marco explicativo de la realidad comunitaria (Campo \& Labarca-Reverol, 2009).

\section{Toda forma de violencia de género nace a partir de la no valoración de las mujeres en el territorio}

En la primera representación social se configura como núcleo central la "no valoración del papel de la mujer en el territorio", siendo este el elemento que determina la estructura de la representación y le otorga un significado de acuerdo con las condiciones históricas, sociales e ideológicas relacionadas con los valores y las normas compartidas por la comunidad. Así pues, para las mujeres indígenas del territorio Cañamomo Lomaprieta, la violencia está determinada por la desvaloración de su trabajo y su rol en el territorio, lo cual se arraiga en creencias que vinculan el ser mujer con el hogar y la reproducción. Este núcleo central es reforzado por la vivencia de diferentes formas de violencia que se constituyen en los elementos periféricos de la representación social. De esta forma, se hace evidente en el discurso de las mujeres la vivencia de violencia física 
como una expresión de la creencia que asume a la mujer como un ser indefenso y dependiente de los hombres, lo que genera un vínculo asimétrico que pone al hombre en una posición de superioridad frente a la mujer.

En el caso de la violencia verbal-psicológica, se define en el campo de representación asociada a un daño en su estabilidad mental a través de formas de abuso que, en el ámbito privado, se manifiestan en palabras soeces y, en el ámbito social, a través del irrespeto por los elementos propios de su identidad como mujeres indígenas, esto es, sus creencias, su territorio y sus hijos. Esto conlleva que la violencia verbal-psicológica sea reconocida como la más fuerte y representativa, es decir, como aquella que causa más daño, pues desvaloriza a la mujer al no otorgarle la importancia que merece.

Otro elemento periférico es la ocurrencia de violencia económica contra las mujeres, representada en el acceso limitado a educación y trabajo, anclada a la creencia de que la mujer pertenece al hogar y a las labores de cuidado, lo cual tiene un carácter histórico y cultural trasmitido a través de la interacción comunicacional y normalizada socialmente por parte de las mujeres y la comunidad.

En último lugar, se encuentra la violencia sexual, a la cual se le otorga un carácter de desequilibrio de poderes, pues las mujeres conciben que esta se presenta porque los hombres consideran a la mujer menos importante que ellos y, por lo tanto, como un objeto de su propiedad, lo que limita su posibilidad de elegir; en este sentido, la sexualidad en la mujer del territorio es concebida como parte de las labores de cuidado del hogar y el matrimonio, dejando de lado la noción de disfrute y goce sexual.

Por lo dicho, se presenta una primera representación social en la cual la violencia contra la mujer como objeto de representación es significada como la consecuencia de un proceso histórico cultural que ha dado como resultado la no valoración de la mujer en el territorio, al considerarse que su labor fundamental está en el hogar y en el cuidado de la familia, y entenderse en el conocimiento común como algo de la naturaleza femenina las labores de cuidado y reproducción. Esto explica que en la cotidianidad se presenten manifestaciones de violencia física, económica, sexual, verbal y psicológica que se constituyen en el sistema periférico que lo refuerza. Estas manifestaciones son entendidas como acciones utilizadas para reafirmar el poderío masculino e imponer las reglas de comportamiento que las mujeres deben seguir en el ámbito privado-familiar y en los ámbitos públicos, según roles preestablecidos aceptados por las mujeres y la comunidad, que no le otorgan valor a las labores femeninas y derivan en actos que limitan su desarrollo en otros espacios. Esta representación social de la violencia de género permite a las mujeres comprender la realidad vivida de acuerdo a un sistema de valores que las ubica en un rol desvalorizado y se trasfiere culturalmente como una práctica compartida. 


\section{La violencia de género se forja en el hogar}

Como idea principal que sostiene esta representación social aparece la noción de que "los hombres de la comunidad son violentos porque en su hogar vivieron la violencia", concepto a partir del cual se puntualiza la violencia como una conducta aprendida en el seno de la familia a través de pautas educativas y de socialización en las que el maltrato es sufrido u observado por el hombre en su niñez y reproducido en su vida adulta. Este núcleo es reforzado por unos elementos periféricos que aparecen como opiniones derivadas de vivencias propias en las que la educación en el hogar se concibe como una forma de reproducción o prevención del maltrato. Así pues, se identifica que "la violencia se trasmite de padres a hijos" a través de la enseñanza de roles diferenciados para hombres y mujeres y del ejemplo de conductas violentas del padre hacia la madre, lo que confluye en la reproducción de este comportamiento. En relación con este elemento periférico aparece uno segundo, que indica que "se debe educar a los hijos para que no sean otra fuente de agresión", a través del cual las mujeres reconocen en la educación una forma de prevención de la violencia de género. Así pues, plantean la posibilidad de utilizar nuevos patrones de crianza en sus hogares, a fin de generar cambios en las pautas de comportamiento y en las formas de relacionarse hombres y mujeres en pro de que la violencia contra la mujer no se siga presentando en la comunidad.

Se genera, de esta forma, una segunda representación social de la violencia contra la mujer en el territorio, en la que esta se significa como una conducta aprendida y trasmitida en el hogar mediante la observación e imitación de actos violentos efectuados por el padre y la enseñanza de estereotipos y roles que asocian lo masculino con el uso de la fuerza, el dominio o la agresividad. Esta representación parte de experiencias propias y de informaciones del contexto que indican que el maltrato hacia la mujer es trasmitido en las relaciones de padres e hijos, pues se reconoce que en el ámbito familiar es donde tiene mayor ocurrencia; lo anterior, de acuerdo con elementos de la historia de vida de las mujeres y de su contexto inmediato, tales como las pautas educativas con las que fueron educadas y las que en la actualidad sostienen en sus hogares. Los elementos periféricos de la representación han desarrollado un papel fundamental en la adaptación de la misma a la evolución del contexto, puesto que, si bien es cierto que las mujeres reconocen la importancia de educar con modelos más respetuosos que no tomen la violencia como un referente de autoridad, este reconocimiento adquiere un carácter de condicionalidad, pues se reitera que el castigo físico es una forma efectiva de poner límites. Estos elementos de la representación social cumplen la función de generar explicaciones acerca del origen de la violencia y, al mismo tiempo, de orientar las prácticas en los hogares, posibilitando así que en la comprensión de la realidad el maltrato sea entendido como algo trasmitido intergeneracionalmente. 


\section{Ante la violencia de género contra la mujer en el territorio, es más oportuno quedarse callada}

En la tercera representación social se presenta como categoría repetida a través de las 3 dimensiones de análisis de la representación la noción nuclear de que "intervenir en defensa de las mujeres cuando son violentadas es meterse en problemas". Esta creencia muestra gran relevancia y significación dentro del sistema estructural de la representación, configurándose como el núcleo central por cuanto permite explicar un sistema de valores sociales que derivan en una actitud de indiferencia ante los casos de violencia en la comunidad, determinada por informaciones que caracterizan a la familia y a la pareja como un espacio privado en el que no cabe la intervención de terceros y que al ser transgredido deriva en problemas que ponen en riesgo la integridad física y social de quien transgrede. Reforzando este núcleo se reconocen 4 concepciones.

La primera de ellas señala que "los problemas de pareja son privados y nadie debe meterse en ellos", lo que indica que, según los valores de la comunidad, es un deber respetar el espacio familiar como algo propio, basado en la actitud de defender la intimidad del hogar; este elemento periférico conlleva que las situaciones de maltrato contra la mujer sean obviadas o definidas como una problemática que la mujer o la familia deben solucionar.

La segunda concepción que aparece en el campo de esta representación es que "la pareja se arregla y uno queda con enemigos", lo cual refuerza la idea de no intervenir en ayuda de la mujer violentada, basado en una opinión generalizada que sostiene que la pareja es capaz de solucionar un problema de violencia sin ayuda o intervención de terceros, y que la denuncia de personas ajenas al problema conlleva que el denunciante se gane problemas innecesarios con sus vecinos. De manera similar, las mujeres afirman que los problemas de violencia se solucionan en pareja, de manera privada y en la cama, lo que indica una significación de la sexualidad como un atenuante de la violencia.

La tercera concepción revela que "si uno cuenta queda como un chismoso y arriesga su propia vida", lo cual identifica la necesidad de salvaguardar la propia imagen e integridad por encima de la de otras personas, actitud reforzada por sentimientos de miedo e impotencia que orientan conductas indiferentes y de silencio ante los casos de violencia contra la mujer.

La cuarta y última concepción responde a la idea de que "intervenir significa obtener problemas con la misma mujer afectada, pues ella defiende a su agresor"; esta concepción nace de experiencias comunitarias que han sido trasmitidas como un conocimiento práctico a través de la comunicación y la interacción social. Así pues, se describen situaciones en las que la autoridad intenta intervenir y la mujer agredida sale en defensa de su pareja, lo cual es reforzado por la noción 
de una mujer dependiente emocional y económicamente de la figura masculina en el hogar, con bajo nivel educativo, acostumbrada a la violencia y con conductas o expresiones que justifican el comportamiento de su agresor.

\section{Las mujeres que son permisivas con la violencia son culpables de esta}

"Si la mujer es permisiva es culpable de la violencia", este es el núcleo que sostiene la cuarta y última representación social encontrada, explicando una serie de creencias que, basadas en un reconocimiento de la importancia de la igualdad de derechos, critican la actitud de las mujeres víctimas frente a la situación de violencia de género, señalando que el maltrato se da a causa de su permisividad y que, por lo tanto, son culpables del mismo. Adicionalmente, se genera una serie de explicaciones que imponen a la mujer víctima la función de cuidarse y prever la violencia.

Reforzando este núcleo, se presenta la creencia de que la mujer afectada es la encargada de poner reglas en la relación, lo que fortalece patrones de conducta social que imponen a la mujer la responsabilidad de resistirse ante el maltrato bajo la norma cultural que supone que la mujer es la responsable de garantizar el funcionamiento armónico de la relación de pareja o del grupo familiar. Esto implica que, para la mujer víctima de violencia de género, sufrir maltrato conlleve para sí sentimientos de vergüenza y auto culpabilidad, lo cual se convierte en uno de los elementos que obstaculiza la denuncia y el abandono de una relación violenta. Aunado a este pensamiento, se define el siguiente elemento periférico, que pone de manifiesto que pedir ayuda es responsabilidad de la mujer afectada, lo que ubica a la denuncia como el elemento más inmediato y eficaz para salir de la situación de violencia. De ahí que este elemento periférico otorgue a la víctima el deber de pedir ayuda, quien, si no lo hace, representa una mujer con poca capacidad de decisión y con escasa calidad moral puesto que transgrede la regla social de solicitar ayuda, siendo regularmente señalada como tonta y permisiva.

Esta representación social de la víctima como una mujer permisiva indica la existencia de estereotipos a través de los cuales se responsabiliza a las víctimas por sufrir el maltrato. Así, por ejemplo, en el campo de la representación aparecen contenidos que asocian la figura de la víctima con la de una persona sumisa, dependiente, con pocos valores morales, con baja autoestima y con problemas mentales. Estas características, que aparecen de manera generalizada en las verbalizaciones de las mujeres, indican una postura que acusa a la mujer por el comportamiento asumido frente a la violencia, señalando que ella es responsable de poner reglas en la relación a fin de prevenir el maltrato y quien, en caso de ser víctima, debe pedir ayuda a las autoridades. De lo contrario, esa actitud señalada como pasiva la hace responsable de la violencia. 
Expresiones como "el hombre llega hasta donde la mujer lo permite", presentes en las declaraciones de las mujeres sujeto de estudio, permiten que se mantenga la creencia histórica de que la mujer es quien debe prevenir la violencia, y deriva en un señalamiento social hacia la víctima, lo que, a su vez, obstaculiza la denuncia. Del mismo modo, se reconoce en la declaración de las mujeres el pensamiento que indica que la mujer es la encargada de mantener el bienestar familiar, lo cual conlleva que se presente un sentimiento de culpa en la víctima, que refuerza la no denuncia de la situación, puesto que entra en juego la imagen social de la mujer al considerarse la posibilidad de ser señalada como provocadora o culpable del maltrato. Así, la representación social determina el conocimiento de lo que es socialmente aceptado en el territorio y guía el actuar de las mujeres.

\section{Discusión}

La violencia contra las mujeres indígenas debe ser entendida en constante interacción con los elementos propios de su identidad (FIMI, 2006); en este sentido, es necesario vislumbrar el origen de las prácticas que, enmarcadas en una cultura propia, la permiten y mantienen en un territorio y contexto dado, para lo cual se reconoce en la teoría de las representaciones sociales un marco teórico que permite identificar aquellos elementos de la cultura que al ser trasmitidos históricamente podrían consentir la permanencia de la violencia contra la mujer. Siguiendo este planteamiento, en la presente investigación se buscó comprender el fenómeno de la violencia de género al interior del territorio a través del estudio de las representaciones sociales. Los resultados del estudio arrojan una serie de representaciones vinculadas principalmente a la trasmisión de roles, que ponen a las mujeres en una situación de desventaja social frente a los hombres.

A este respecto, se considera importante tener presente la posición de la historiadora feminista Joan W. Scott (1999), quien considera al género como un ordenador social y una forma primaria de los significantes de las relaciones de poder, haciendo alusión principalmente a los símbolos culturalmente disponibles que evocan representaciones múltiples y contradictorias y a los conceptos normativos como interpretaciones de los significados de esos símbolos, y señalando que las estructuras jerárquicas se basan en la comprensión generalizada de la relación natural entre hombre y mujer. En palabras de Estrella-Vega (2018), esto permite la subordinación justificada en esa diferencia que exacerba situaciones de violencia y legitima un sistema de dominación que define en el contexto específico el lugar que ocupan las mujeres. Estas premisas se evidencian en los resultados del estudio, donde los campos de representación identificados dan cuenta de la hegemonía del género masculino sobre el femenino, y de cómo el conjunto de roles de las mujeres se ordena culturalmente de acuerdo a un marco normativo social explícitamente patriarcal. 
Los resultados de este estudio evidencian una primera representación social estructurada a partir de un núcleo que considera la violencia como una consecuencia de la no valoración de la mujer en el territorio, lo que se relaciona con una condición de menor importancia en el sistema de dominación-subordinación del que habla Scott (1999), anclada a una creencia histórica que vincula lo femenino a una labor de cuidado y reproducción -como función natural del ser mujer- y que deriva en manifestaciones de violencia física, violencia verbal-psicológica, violencia sexual y violencia económica. Con esta consideración, los hallazgos de Cediel-Becerra et al. (2017) concuerdan con los del presente estudio, al afirmar que la mujer está generalmente expuesta a mayores desigualdades y violencias debido a que su valoración social es menor que la del hombre, y que la violencia de género como forma de discriminación es causada por prejuicios sociales arraigados en la división sexual del trabajo (Meza-Martínez, 2018), lo que está asociado con las representaciones sociales preferentemente heteronormativas.

En el contexto territorial, estas apreciaciones se manifiestan en el imperativo cultural que relega un $75 \%$ de las mujeres al ámbito doméstico (AMICL, 2017), que al ser transgredido expone a las mujeres a diversas formas de violencia, según lo relacionan en el campo periférico de la representación social. Esto, en palabras de Segato (2018), se puede entender como una forma de disciplinar y despojar a las mujeres de los medios de existencia y capacidades sociales y políticas, expandiendo y sosteniendo cotidianamente la concepción de superioridad de lo masculino. Se entiende, al respecto, que el problema de la violencia de género contra la mujer radica en que la situación de discriminación de la mujer está fundamentada en concepciones, normas sociales, valores y estereotipos que se encuentran arraigados en la cultura y exponen a las mujeres a múltiples y particulares formas de violencia que se esconden en representaciones sociales orientadas a la designación de roles que las someten, de una forma socialmente aceptada, a labores domésticas, de servicio, de cuidado, de reproducción, de represión, manifiestas en usos abusivos de sus cuerpos, tiempo, necesidades y ejercicio pleno y autónomo de su libertad en todas las esferas (Alta Consejería Presidencial, 2012).

Estos valores socioculturales nacen a partir de la interiorización de los roles, estereotipos y conductas establecidas en el seno de las familias como agentes de transmisión cultural y de normalización de la violencia de género como norma social. De acuerdo con Echeburúa y Del Corral (2004), el maltrato es un comportamiento aprendido a través de la imitación de patrones de violencia repetitivitos ejercidos por el varón (padre) hacia la mujer (madre), que se significan como un recurso eficaz y aceptable para enfrentarse a las frustraciones del hogar, lo que conlleva a la reproducción de la violencia en las siguientes generaciones, tal como puede evidenciarse en la representación social "la violencia de género se forja en el hogar"; lo anterior fundamenta la estructura de una representación que apunta a la familia como principal generador de pautas de maltrato, a través de modelos educativos que legitiman la agresión como ejercicio de autoridad y poder, y reiteran la diferenciación de roles de acuerdo al sexo. En contraste con lo anterior, Varela 
(2016) indica que los hijos de maltratadores probablemente se conviertan en su vida adulta en victimarios, puesto que al interiorizar los roles de género naturalizan la violencia como una forma de relacionarse entre hombres y mujeres.

En ese escenario, Alfocea-Frutos y Ponce-Alcaraz (2019) invitan a profundizar mayormente en la infancia de la víctima, pues indican que las mujeres que provienen de hogares en los que se ha ejercido la violencia de género tienden a asimilar roles y estereotipos determinados que normalizan en su vida la desigualdad entre hombres y mujeres, justificando el comportamiento del hombre en un rol de poder y masculinidad. En este sentido, se debe tomar en cuenta la noción de que la violencia de género en el resguardo indígena podría prevenirse en el hogar bajo la premisa de que "se debe educar a los hijos para que en el futuro no sean otra fuente de agresión", lo que implica la transformación de los modelos de crianza bajo los cuales fueron educadas las mujeres. Este elemento periférico es fundamental en el desarrollo de programas para la prevención de la violencia en el resguardo, entre tanto puede presentarse como un punto de inflexión en la representación social donde la familia, en cuanto institución social, se encarga de trasmitir las normas, valores y modelos de identidad, de acuerdo con una nueva forma de organización familiar que deconstruya la convencional estructura asimétrica entre hombres y mujeres (Martínez-García \& Camarero, 2015).

En este sentido, las formas convencionales y normativas de organización familiar se pueden entender como un espacio en el que la violencia se promueve, puesto que en el territorio se encuentran estructuras familiares jerarquizadas que al ser concebidas como espacios privados que no deben ser transgredidos por terceras personas derivan en una representación social fundamentada en la creencia de que la intervención en favor de mujeres agredidas es sinónimo de obtener problemas con los implicados, pues se sobrepone la privacidad del hogar sobre los derechos individuales de la víctima. Al respecto, Yugueros-García (2014) explica esta situación como una consecuencia del pensamiento social que considera las relaciones de pareja como un asunto de dominio privado y que, por lo tanto, incita a tomar una actitud silenciosa y permisiva frente a prácticas de maltrato o violencia, a pesar de su conocimiento; lo que coincide con Rico (1996), quien reconoce que en la relaciones de pareja la privacidad actúa como factor de orden ideológico-cultural que inhibe a la comunidad para la toma de decisiones y disminuye la frecuencia de denuncias vecinales de hecho sobre violencia de género contra la mujer. Gran parte de este dispositivo cultural representa a la violencia como una práctica en el ámbito doméstico, cuya solución queda relegada explícitamente a la gestión de las dinámicas conyugales, que incluso podrían justificarla.

Para Rico (1996), la aceptación y defensa del agresor por parte de la mujer responde a la internalización de los valores sociales que indican la subordinación femenina como algo natural, la aceptación de normas culturales que regulan la vida en pareja y los roles que cada sexo debe tomar, la idealización de la familia y el matrimonio, y las normas sociales que rigen los compor- 
tamientos de acuerdo a la cultura, tal como se observa en los hallazgos del estudio. No obstante, de acuerdo a las representaciones sociales de las mujeres de Cañamomo, su deber como mujeres es formar un hogar bien constituido, por lo tanto, los problemas familiares o de pareja son parte de la intimidad y pueden justificarse mientras permitan el mantenimiento de la estructura familiar. Lo anterior, de acuerdo a Naula y Jácome (2016), guarda una estrecha relación con una violencia de género que ha sido heredada culturalmente en la lógica de una organización familiar que debe ser respetada por encima de cualquier acontecimiento, y que explica los bajos índices de denuncia en la comunidad -que alcanzan solo un $20 \%$, ocultando cifras que, según las autoridades del resguardo, son más altas (AMICL, 2017)-.

Son evidentes en la comunidad de Cañamomo la internalización de los modelos cognitivos de superioridad masculina evidenciados en los discursos de las mujeres, mismos que, de acuerdo a Afocea-Frutos y Ponce-Alcaraz (2019), impiden que estas se perciban a sí mismas y a las demás como víctimas y las hace vulnerables a las distorsiones cognitivas relativas a sus roles, además de normalizar la violencia y llevar a que, a menudo, la mujer se culpe a sí misma por la situación de maltrato. Esta apreciación se hace evidente en la representación social que sostiene que las mujeres que permiten el maltrato son culpables del mismo, postura que responsabiliza a la víctima sobre el hecho de violencia y dificulta la denuncia; hallazgo confirmado por Gómez (2016), quien destaca que las mujeres que son víctimas de violencia de género han sido tradicionalmente culpadas, adjudicándoles estereotipos negativos debido a que la denuncia es interpretada por la sociedad como la salida legítima de la violencia, lo que responsabiliza a la mujer que no es capaz de denunciar y la señala de sumisa, boba o masoquista. En esta línea, Deza-Villanueva (2012) señala que el juicio social que se establece frente a las víctimas por no denunciar su situación agrava su aislamiento y dificulta romper el ciclo de maltrato, puesto que aceptan la culpa de su sufrimiento, reforzando la desvalorización provocada por el agresor y la situación de dependencia, hecho que, de acuerdo a Izquierdo (2013), responde a mitos e imaginarios sociales con los que se intentan explicar las causales de la violencia de género y se justifica indirectamente al agresor al responsabilizar a la víctima de los abusos sufridos.

En relación con estudios encontrados sobre representaciones sociales de violencia contra las mujeres, la mayor parte de ellos coinciden con los hallazgos en aspectos como la percepción de permisividad de las mujeres en la violencia, la génesis de la violencia en el hogar, la tendencia de las mujeres a defender a sus agresores, la atribución de que los hijos varones que han presenciado la violencia contra sus madres tienden en su vida adulta a reproducir contra las mujeres la violencia observada (Morais del Río, 2018; Bouroncle, 2016; Sánchez, 2019; Pérez \& Argumedo, 2017). También se encuentran algunas diferencias con Morais del Rio (2018), quien sostiene que las representaciones sociales de la violencia de género no se vinculan con el desequilibrio de poder entre hombres y mujeres, caso contrario a los resultados del estudio, donde para las mujeres de Cañamomo toda forma de violencia de género se explica desde prácticas discriminatorias que llevan a su no valoración en el territorio; también discrepa en el análisis de la tipificación de las 
violencias ejercidas, entre tanto concibe que es la física la que tiene mayor recurrencia, mientras las mujeres de Cañamomo reconocen a la violencia psicológica como la más fuerte y la asocian, además, al irrespeto por sus creencias. No se encuentran coincidencias sobre la representación social que indica que denunciar es meterse en problemas, como tampoco estudios que explícitamente aborden las representaciones sociales en mujeres indígenas.

Finalmente, el estudio de las representaciones sociales permite comprender la violencia de género contra la mujer en el caso de las mujeres indígenas del resguardo Cañamomo Lomaprieta respecto del reconocimiento de roles tradicionales que permitan deconstruir el papel que ocupan las mujeres en sus territorios y las normas y valores sociales que sostienen prácticas patriarcales desde las cuales se sustentan las creencias que determinan la tendencia a responsabilizar a la mujer del maltrato y perpetúan, a su vez, una condición de desigualdad inmanente al género desde donde se mantienen los nichos intergeneracionales de violencia contra las mujeres (Ferrer-Pérez et al., 2006).

\section{Conclusiones}

El concepto de violencia de género en el contexto indígena debe construirse necesariamente teniendo como referente las miradas y representaciones de quienes la vivencian, pues su carácter y concepción dependerá de la interacción entre los factores sociales, políticos, culturales e históricos propios del contexto. En efecto, el estudio de la violencia de género debe adquirir un enfoque que posibilite un análisis más profundo de los elementos diferenciales que, en interacción, incrementan la desigualdad y derivan en formas particulares de vivir y entender las situaciones de opresión entre géneros históricamente asimétricos.

En el caso de las mujeres del resguardo indígena Cañamomo Lomaprieta, la violencia contra la mujer ha sido significada y vivida de acuerdo con los valores históricos, sociales y culturales que han sido trasmitidos de generación en generación a través de las pautas de crianza, el intercambio comunicacional y las diversas formas de interacción social. Esto da como resultado un conocimiento común que posiciona a las mujeres como inferiores frente a los hombres, lo que en las prácticas individuales y colectivas se traduce en comportamientos de indiferencia o silencio social ante los casos de maltrato y lleva a la culpabilización de la mujer al considerarla permisiva con la violencia. Esta lectura de la violencia contra la mujer en la comunidad permite reflexionar acerca de la necesidad de generar estrategias integrales que, desde un enfoque holístico e interseccional, respondan a la violencia de género, entendiendo las representaciones sociales que la 
mantienen y sus elementos constitutivos como núcleo de las prácticas y, por lo tanto, como una guía que permita entrever las relaciones histórico culturales y los fenómenos sociales asociados a la reproducción de la violencia.

\section{Conflicto de intereses}

Los autores declaran la inexistencia de conflicto de interés con institución o asociación comercial de cualquier índole.

\section{Referencias}

Abric, J. (2001). Prácticas sociales y representaciones. Ediciones Coyoacán.

Alfocea-Frutos, J., \& Ponce-Alcaraz, F. J. (2019). Perspectiva criminológica sobre la violencia de género. La razón histórica, 43, 104-121. https://dialnet.unirioja.es/servlet/ articulo?codigo $=6964673$

Alta Consejería Presidencial. (2012). Lineamientos de la política pública nacional de equidad de género para las mujeres. Bogotá Colombia. http://www.equidadmujer.gov.co/ejes/Documents/Lineamientos-Politica-Publica-Equidad-Genero.PDF

Asociación de Mujeres del Resguardo Indígena Cañamomo Lomaprieta del Municipio de Riosucio Caldas (AMICL). (2017). Caracterización para el fortalecimiento del proceso organizativo de las mujeres del resguardo indígena Cañamomo Lomaprieta.

Barragán, F., \& Alfonso, M. (2010). Lineamientos de política pública para la prevención, protección, atención y sanción de las violencias basadas en género y contra las mujeres en Colombia. Documento de trabajo. https://www.minsalud.gov.co/sites/rid/Lists/BibliotecaDigital/RIDE/INEC/IGUB/Lineamientos-politica-publica-violencia-mujeres.pdf

Barragán (2015). Cartografía social pedagógica: entre teoría y metodología. Revista Colombiana de Educación, 70, 247-285. http://www.scielo.org.co/pdf/rcde/n70/n70a12.pdf 
Bouroncle, S. (2016). Representaciones sociales de violencia contra las mujeres en autoridades de un distrito rural costero [Tesis de Licenciatura, Pontificia Universidad Católica del Perú]. Repositorio PUCP. http://hdl.handle.net/20.500.12404/7272

Campo, M., \& Labarca-Reverol, C. (2009). La teoría fundamentada en el estudio empírico de las representaciones sociales: un caso sobre el rol orientador del docente. Opción, 25(60), 4154. https://www.redalyc.org/pdf/310/31012531004.pdf

Ceballos, F., \& González, B. (2018). Representaciones sociales sobre el maltrato que ejerce el cliente a trabajadores de una empresa transnacional de combustible. Revista Sul Americana de Psicología, 4(2), 240-272. http://ediciones.ucsh.cl/ojs/index.php/RSAP/article/ view/1785

Cediel-Becerra, N. M., Hernández-Manzanera, J., López-Duarte, M. C., Herrera-Buitrago, P., Donoso-Burbano, N., \& Moreno-González, C. (2017). Empoderamiento de las mujeres rurales como gestoras de los Objetivos de Desarrollo Sostenible en el posconflicto colombiano. Equidad y Desarrollo, (28), 61-84. https://doi.org/10.19052/ed.4077

Comisión Interamericana de Derechos Humanos (CIDH). (2017). Las mujeres indígenas y sus derechos humanos en las Américas. (Doc. 44/17) http://www.oas.org/es/cidh/informes/ pdfs/mujeresindigenas.pdf

Congreso Nacional de la República de Colombia. (2008). Ley 1257. Por la cual se dictan normas de sensibilización, prevención y sanción de formas de violencia y discriminación contra las mujeres. https://www.oas.org/dil/esp/ley_1257_de_2008_colombia.pdf

Cuevas, Y. (2016). Recomendaciones para el estudio de representaciones sociales en investigación educativa. Cultura y Representaciones Sociales, 11(21), 109-140. http://www.scielo. org.mx/pdf/crs/v11n21/2007-8110-crs-11-21-00109.pdf

Deza-Villanueva, S. (2012). ¿Por qué las mujeres permanecen en relaciones de violencia? Avances en Psicología, 20(1), 45-55. https://doi.org/10.33539/avpsicol.2012.v20n1.1942

Echeburúa, E., \& Del Corral, P. (2004). Violencia doméstica: ¿Es el agresor un enfermo? Formación Médica Continuada en Atención Primaria, 11(6), 293-299. https://doi.org/10.1016/ S1134-2072(04)76123-X

Estrella-Vega, M. (2018). Entre la autonomía y la subordinación: significados y perspectivas de la experiencia migratoria de mujeres centroamericanas en tránsito por México. Sociológica, 93, 247-280. http://www.scielo.org.mx/scielo.php?script=sci_abstract\&pid=S0187$01732018000100247 \& \operatorname{lng}=$ es\&nrm $=$ iso 
Ferrer-Pérez, V., Bosch-Fiol, E., Ramis-Palmer, C. y Navarro-Guzmán, C. (2006). Las creencias y actitudes sobre la violencia contra las mujeres en la pareja: Determinantes sociodemográficos, familiares y formativos. Anales de Psicología, 22(2), 251-259. https://revistas. um.es/analesps/article/view/25901

Foro Internacional de Mujeres Indígenas (FIMI). (2006). MAIRIN IWANKA RAYA. Mujeres indígenas confrontan la violencia. Informe complementario al estudio sobre violencia contra las mujeres del secretario general de las naciones unidas. http://www.fimi-iiwf.org/archi vos/8162f56478b843333dc95a1f5f381ab1.pdf

Glaser-Holton, B. G. (2004). Remodeling Grounded Theory. Forum Qualitative Social Research, 5(2), 23-32. https://doi.org/10.17169/fqs-5.2.607

Gómez, E. (2016). Culpabilización de las víctimas y reconocimiento: límites del discurso mediático sobre la violencia de género. Feminismo/s, 197-218. https://rua.ua.es/dspace/bitstream/10045/61254/1/Feminismos_27_11.pdf

Hamui, A. y Varela, M. (2013). La técnica de grupos focales. Metodología de investigación en educación médica. Investigación en Educación Médica, 2(1), 55-60. https://doi.org/10.1016/ S2007-5057(13)72683-8

Izquierdo, A. (2013). Desmontando mitos: La violencia de género durante el embarazo [Tesis de Maestría, Universidad de Salamanca]. https:/gredos.usal.es/bitstream/hand1e/10366/122420/TFM_IzquierdoMoreta_Desmontando.pdf;jsessionid=BEA26ECE5C1 7EF7FE0B48F5683F697E1? sequence $=1$

Juárez-Moreno, M., Raesfeld, L. J. y Durán-González, R. E. (2021). Diagnóstico interseccional de violencia hacia mujeres indígenas. Revista Estudios Feministas, Florianópolis, 29(1), 1-11. https://doi.org/10.1590/1806-9584-2021v29n163207

Martínez-García, M. A. y Camarero, L. A. (2015). La reproducción de la violencia de género: una lectura desde las áreas rurales. Ager. Revista de Estudios sobre Despoblación y Desarrollo Rural, 19, 1-30. https:/www.redalyc.org/pdf/296/29642953004.pdf

Meza-Martínez, C. A. (2018). Discriminación laboral por género: una mirada desde el efecto techo de cristal. Equidad y Desarrollo, (32), 11-31. https://doi.org/10.19052/ed.5243

Mora, M. (2002). La teoría de las representaciones sociales de Serge Moscovici. Athenea digital, (2), 1-25. https://www.raco.cat/index.php/Athenea/article/viewFile/34106/33945 
Morais del Río, M. (2018). Representaciones sociales de la violencia de género en un grupo de mujeres de Centro Habana. Revista Sexología y Sociedad, 24(1), 34-44. http://revsexologiaysociedad.sld.cu/index.php/sexologiaysociedad/article/view/639/661

Naula, G. L. y Jácome, M. D. (2016). Conocimientos, actitudes y prácticas sobre la violencia de género de la población indígena, atendida en el hospital Asdrubal de la torre del Cantón Cotacachi, durante el periodo abril-agosto 2016 [Tesis Especialización, Pontificia Universidad Católica del Ecuador]. Repositorio de tesis de grado y posgrado. http://repositorio.puce.edu.ec/handle/22000/12671

Nuño, B. (2004). Modelo de toma de decisiones con los que intentan resolver el consumo de drogas ilegales adolescentes consumidores y sus padres que acuden a tratamiento a CIJ en Guadalajara [Tesis doctoral, Universidad Nacional Autónoma de México]. Dirección General de Bibliotecas. http://132.248.9.195/ppt2004/0334319/0334319.pdf

Ortiz, L. (2012). Curso de investigación cualitativa. Universidad Nacional Abierta y a Distancia$U N A D$. Escuela de Ciencias Sociales Artes y Humanidades.

Pérez, F., \& Argumedo, D. (2017). Representaciones sociales de violencia contra las mujeres en pobladoras de un distrito rural costero [Tesis de licenciatura, Pontificia Universidad Católica del Perú]. Repositorio PUCP. http://hdl.handle.net/20.500.12404/9309

Portilla, M., Rojas, F. A., \& Hernández, I. (2014). Investigación cualitativa: una reflexión desde la educación. Universitaria: Docencia, Investigación e Innovación, 3(2), 86-100. https:// revistas.udenar.edu.co/index.php/duniversitaria/article/view/2192

Quintero, L., \& García, L. E. (2021). Mutilación genital femenina en perspectiva forense como violencia de género. Revista Eleuthera, 23(1), 83-97. https://revistasojs.ucaldas.edu.co/ index.php/eleuthera/article/view/4227/3889

Rateau, P., \& Mónaco, G. (2013). La teoría de las representaciones sociales: orientaciones conceptuales, campos de aplicaciones y método. CES Psicología 6(1), 22-42. http://www. scielo.org.co/scielo.php?pid=S2011-30802013000100003\&script=sci_abstract\&tlng=es

Restrepo, D. (2013). La teoría fundamentada como metodología para la integración del análisis procesual y estructural en la investigación de las representaciones sociales. Revista CES Psicología, 6(1), 122-133. https://revistas.ces.edu.co/index.php/psicologia/article/ view/2579/1824 
Rico, N. (1996). Violencia de género: un problema de derechos humanos. Documento de la CEPAL, serie mujer y desarrollo. http://hdl.handle.net/11362/5855

Sánchez, S. (2019). Representaciones sociales sobre la violencia de género en policías de Lima Metropolitana [Tesis de licenciatura, Pontificia Universidad Católica del Perú]. Repositorio PUCP. https://tesis.pucp.edu.pe/repositorio/bitstream/handle/20.500.12404/15782/Sanchez_Carrasco_Representaciones_sociales_violencia1.pdf? sequence $=1 \&$ isAllowed $=y$

Scott, W. J. (1999). El género: una categoría útil para el análisis histórico. En M. Navarro y C. R. Stimpson (Comps.), Sexualidad, género y roles sexuales (pp. 265-302). Fondo de Cultura Económica.

Segato, R. L. (2018). Contra-pedagogías de la crueldad. Prometeo Libros.

Varela, N. (2016). Barcos y corazones. Las sutilezas del patriarcado en la transmisión de valores que alimentan la violencia de género. Revista Metamorfosis, (4), 59-73.

Yugueros-García, A. (2014). La violencia contra las mujeres: conceptos y causas. Barataria Revista Castellano-Manchega de Ciencias Sociales, (18), 147-159. https://doi.org/10.20932/ barataria.v0i18.49 